\title{
Assessment and follow up of depressed patients by practice nurses was similar to care by GPs
}

\author{
Mann AH, Blizard R, Murray J, et al. An evaluation of practice nurses working with general practitioners to treat people with \\ depression. BrJ Gen Pract 1998 Jan;48:875-9.
}

\section{Question}

What extended role can practice nurses have in the assessment and follow up of depressed patients who are seen by general practitioners (GPs)?

\section{Design}

2 concurrent 4 month randomised controlled trials.

\section{Setting}

20 general practices in the UK.

\section{Patients}

577 patients (mean age 46 y, $78 \%$ women) whose GPs considered them to have been depressed for $\geqslant 4$ weeks. Exclusion criteria were suicidal ideation, manic depressive psychosis, or current treatment for depression from specialist psychiatric services. Follow up was $88 \%$ (study 1 ) and 92\% (study 2).

\section{Intervention}

All patients received a standardised psychiatric assessment by nurses and completed the Beck Depression Inventory (BDI). In study 1, nurses reported the psychiatric assessment results to GPs and provided a summary in the notes for 74 patients. For 82 patients, the psychiatric assessment results were not reported to GPs, but nurses could advise patients to report any specific problems to their GP. In study 2, nurses discussed each patient with the GP who then determined the course of treatment. Patients were allocated to receive follow up from nurses which comprised a recommended 8 hours of contact for each patient $(n=271)$ or standard care from a GP $(n=148)$. Patients who were followed up by nurses could see their GP upon request.

\section{Main outcome measures}

Changes in BDI scores and in the proportion of patients who met the DSM-III criteria for major depression.

\section{Main results}

All groups showed improvement at 4 months. For patients whose psychiatric assessment results were reported to GPs in study 1 , the mean decrease in BDI scores was 7.1 and $59 \%$ fewer patients met DSM-III criteria for major depression. For patients whose assessments were not reported to GPs, BDI scores decreased by 6.9 and $54 \%$ fewer patients met DSM-III criteria for major depression. Study 1 had $<75 \%$ power to detect a $20 \%$ difference at the $5 \%$ level of significance. In study 2, patients seen by nurses had a mean decrease of 10.3 in BDI scores and a $49 \%$ decrease in the proportion of patients who met DSM-III criteria for major depression. For patients seen by GPs, mean BDI scores decreased by 10.6 and $59 \%$ fewer patients met $D S M-I I I$ criteria for major depression. Study 2 had $>90 \%$ power to detect a $20 \%$ difference at the $5 \%$ level of significance. In both studies treatment groups did not differ for mean BDI scores or in the proportion of patients who were depressed.

\section{Conclusions}

Depressed patients whose psychiatric assessment results were reported to general practitioners (GPs) by nurses had similar improved outcomes as those whose psychiatric assessment results were not reported to GPs. Depressed patients who received follow up care from nurses had similar improved outcomes as those who received standard care from GPs.

Source of funding: UK Department of Health.

For correspondence: Professor A H Mann, Department of Psychiatry, Section of Epidemiology and General Practice, The Maudsley Institute of Psychiatry, De Crespigny Park, Denmark Hill, London SE5 8AF, UK. Fax +44 (0)171 2770283.

\section{Commentary}

The first question to ask of a "negative" result is whether the trial was large enough to find a clinically important difference. The report by Mann et al gives power calculations that show a $20 \%$ difference in outcome was assumed, which corresponds to a number needed to treat of about 5 (ie, 5 people would need to be assessed and followed up by nurses to achieve 1 additional good outcome). This effect size was perhaps a bit ambitious for a study that was not investigating a specific intervention.

The patients were those identified by and referred from GPs and they therefore reflect clinical practice. However, the low recruitment rate suggests that some GPs may have been selective when referring patients, although one would not expect this to affect the validity of the conclu- sions. Could this common problem in randomised controlled trials affect the generalisability of the study?

The structured assessment on its own might have had a therapeutic effect. There might also have been an improvement in the care of the "treatment as usual" control group in study 1 . The GP would have received feedback from the nurse on some patients and the structured approach might have altered the GPs' care of patients in the control group. Any effect on compliance was not reported, although prescription rates increased in some groups. The lack of efficacy of the unstructured follow up by nurses in study 2 supports the view that unstructured time with patients does not affect outcome $^{1}$ compared with structured psychotherapies. $^{2}$
The conclusion from this study is that there is still no evidence to support the setting up of clinics in which practice nurses provide follow up for depressed patients. Despite these disappointing results, it seems premature to abandon this interesting idea entirely. Practice nurses have developed an extended role in diabetes and asthma care. They may still have a promising future in the treatment of neurotic disorder.

Glyn Lewis, $\mathrm{PhD}$ Division of Psychological Medicine University of Wales College of Medicine Cardiff, UK

\footnotetext{
1 Friedli K, King MB, Lloyd M, et al. Lancet 1997;350:1662-5.

2 Mynors-Wallis LM, Gath DH, Lloyd-Thomas AK, et al. BMJ 1995;310:441-5.
} 